\title{
Preface to Second Group of "Waves in Plasma" Papers
}

The previous issue of Radio Science contains a group of papers dealing with the subject of "Waves in Plasma." The present issue contains nine more papers on this general topic, which were solicited by the editor.

The first paper by Gross and Felsen presents a systematic theoretical analysis of wave propagation in nonuniform anisotropic media. The authors introduce a novel representation for the $E$ and $H$ modes which results in a type of coupling which is independent of the ionization gradient. The method appears to be particularly suitable for studying regions with strong inhomogeneities along the gyrotropic axis.

The paper by Lee, Peters, and Walter deals with the application of geometrical optic concepts to anisotropic media with nonparallel boundaries. The method considers multiple reflections and related phenomena which are usually neglected in previous studies of this kind.

The following three papers (Akasofu, Megill, and Hines), deal with various aspects of waves in ionospheric type plasma. The papers by Burgess; McLane and Tsukishima are concerned with fluctuation phenomena in plasma and hydromagnetic waves in laboratory plasma, respectively. The following paper by Deschamps shows the advantages of employing graphical technique to give insight to propagation in anisotropic media. The final paper by Tai is a fundamental study of waves in moving anisotropic media.

It is planned that the next issue of the journal will contain a third grouping of papers on "Waves in Plasma."

JAMES R. WAIT, Editor 